\title{
Swiss Experience in Therapy With Dual Bronchodilation in Chronic Obstructive Pulmonary Disease in Relation to Self-Reported Physical Functionality
}

\author{
Marc Spielmanns ${ }^{\mathrm{a}, \mathrm{b}, \mathrm{f}}$, Michael Tamm ${ }^{\mathrm{c}}$, Sebastian Schildge ${ }^{\mathrm{d}}$, Arschang Valipour ${ }^{\mathrm{e}}$
}

\begin{abstract}
Background: Medical therapy in chronic obstructive pulmonary disease (COPD) usually includes inhaled dual bronchodilation leading not only to an improvement in symptoms but also to an increase in physical performance. However, it remains unknown whether responder rates to dual bronchodilation differ between the Swiss subgroup in comparison to participants of other European countries.

Methods: The non-interventional OTIVACTO trial investigated changes in self-reported physical functioning in COPD patients treated with tiotropium/olodaterol $5 / 5 \mu \mathrm{g}$ fixed dose combination for 6 weeks. The cut-off between responder and non-responder was defined as a minimum increase of 10 points using the 10-question physical functioning questionnaire (PF-10) score. We searched for patterns of the responder patient group and compared the results of the whole dataset with the subgroup of Swiss participants.
\end{abstract}

Results: Compared to the total cohort $(n=7,608)$, the Swiss participants $(n=94)$ were predominantly $>65$ years of age and had significantly more comorbidities. There were no significant differences according to COPD stage, smoking status, exacerbation rate in the last 12 months and modified Medical Research Council questionnaire (mMRC) score between the total cohort and the Swiss collective. There were no significant differences between the Swiss subgroup and the total cohort with regard to response to the medication in the PF-10 score. In the intragroup comparison, patients with high mMRC score showed significantly higher values in the PF-10 in both groups.

Manuscript submitted June 17, 2021, accepted June 30, 2021

Published online July 28, 2021

aPulmonary Medicine, Zuercher RehaZentren Klinik Wald, Switzerland

${ }^{b}$ Faculty of Health, Department of Pneumology, University of Witten/Herdecke, Germany

'Lung Centre/Pneumology Department, University Hospital Basel, Basel, Switzerland

${ }^{\mathrm{d}}$ Medical Affairs, Boehringer Ingelheim, Basel, Switzerland

eDepartment of Respiratory and Critical Care Medicine, Karl-LandsteinerInstitute for Lung Research and Pulmonary Oncology, Klinik Floridsdorf, Vienna, Austria

${ }^{f}$ Corresponding Author: Marc Spielmanns, Pulmonary Medicine and Sleep Medicine Center, Zurcher RehaZentren Klinik Wald, CH-8636 Wald-ZH, Switzerland. Email: spielmanns@me.com

doi: https://doi.org/10.14740/jocmr4542
The number of exacerbations had no influence on the PF-10 score in the Swiss subgroup but in the total cohort.

Conclusion: In terms of age and number of comorbidities, significant differences were found between the overall patient population and the Swiss participants, having no influence on the success of the medication. The patients suffering from increased dyspnea benefited most from tiotropium/olodaterol treatment (Clinical Trials Registry NCT02720757).

Keywords: COPD; Physical functioning; PF-10; Tiotropium bromide; Olodaterol

\section{Introduction}

Chronic obstructive pulmonary disease (COPD) is a common, complex and heterogeneous disease which is accompanied by high morbidity and mortality [1]. In 2017, COPD rose to the third leading cause of death worldwide. Breathlessness and exertional limitation are cardinal manifestations of COPD [2]. Dyspnea level and impaired health status vary significantly [3], but are discussed to be predictors of mortality [4]. Predicting and/or modifying the course of the disease remain currently difficult, and selection of patients with a beneficial response to specific interventions is unsatisfactory.

Guidelines recommend inhaled long-acting muscarinic antagonists (LAMAs) and/or long-acting $\beta 2$-agonists (LABAs) as an important therapy in the treatment of COPD [5]. Clinical trials showed that COPD patients medicated with dual bronchodilator therapy (combination of both agents) had significant improvements in exercise capacity and endurance time compared to placebo $[6,7]$. This effect is caused by a reduction of hyperinflation and airway resistance and additionally by improving of ventilatory muscle efficiency [8]. In this context, several randomized controlled trials have confirmed the benefits of a tiotropium/olodaterol (Tio/Olo) fixed dose therapy on exercise capacity $[7,9]$.

However, non-responsiveness to pharmacologic and nonpharmacologic treatments is a common feature in COPD patients and many suffer from refractory symptoms. Up to now, little is known on why a subgroup of patients are not responding to medical treatment and scarce data exist on real-life settings 
regarding the impact of medical treatment on physical functioning. Recently a real-world observational study was published, showing that the treatment with tiotropium/olodaterol led to an improvement in self-reported physical functioning using the 10 -question physical functioning questionnaire (PF-10) across a wide range of COPD severities in a large sample size [10]. This study included mainly patients from Eastern European countries.

An evaluation of COPD treatment guidelines published in Europe and Russia of the past 7 years showed differences in measures used for stratification of disease severity, consideration of patient phenotypes, criteria for the use of inhaled corticosteroids and recommendations for other medications (e.g., theophylline and mucolytics) in addition to bronchodilators [11]. Additionally, a considerable variation in medical care between geographical regions in Europe with substantial differences in clinical outcomes among the healthcare systems with different guidelines and practices might influence patient management.

Data are limited on clinical outcomes in patients with COPD receiving medical therapy in Swiss clinical practice.

Therefore, we analyzed the OTIVACTO dataset with the aim of understanding the clinical outcomes in the subgroup of Swiss COPD patients treated with inhaled Tio/Olo in comparison to the whole European OTIVACTO data.

\section{Materials and Methods}

The design of the OTIVACTO study has been described in detail previously [10]. In summary, nine European countries (Austria, Czech Republic, Hungary, Israel, Romania, Russian Federation, Slovakia, Slovenia, and Switzerland) enrolled COPD patients consecutively over a period of 1 year and treated them with inhaled Tio/Olo via the SPIOLTO soft-mist inhaler device as clinically indicated and approved.

The inclusion criteria for the OTIVACTO trial were patients aged $\geq 40$ years, with a diagnosis of COPD and requiring long-acting dual bronchodilation (LAMA + LABA) treatment (according to physician-based indication and within the approved tiotropium/olodaterol label). The exclusion criteria included treatment with any LAMA/LABA combination (freeand fixed-dose) up to 6 months prior to the study or contraindications according to the approved Spiolto ${ }^{\circledR}$ label. To avoid double-dosing of an LABA, patients who intended to continue LABA/inhaled corticosteroid (ICS) fixed-doe combination treatment were not included in the OTIVACTO study. Other exclusion criteria included patients for whom further followup was not possible at the enrolling site during the planned study period of approximately 6 weeks; pregnancy and lactation; patients currently listed for lung transplantation; and those participating in any clinical trial or any other non-interventional study of a drug or device [10].

Patients were followed up for an observational period of 6 weeks on average.

Several questionnaires were used during the study; the modified Medical Research Council questionnaire (mMRC) scale was used to assess the degree of breathlessness of the patients before treatment at baseline (visit 1). Furthermore, exacerbation history and post-bronchodilator forced expiratory volume in 1 second (FEV1) values were collected in an electronic case report form to automatically calculate the GOLD stage. The PF-10 consisting of 10 questions was used to evaluate physical functioning at baseline (visit 1) and after 6 weeks of treatment (visit 2). The PF-10 is a subdomain of the validated 36-item Short Form Health Survey (SF-36) quality of life questionnaire representing the extent of experienced restrictions while conducting everyday physical activity [12]. Self-reported measures to assess and quantify the functional status of patients as, i.e., the PF-10 questionnaire, are important tools for clinicians and investigators since quality of life limitations can be assessed by measuring different types of functional activities in a real-world setting.

In order to differentiate responder from non-responder in this cohort, a difference threshold of 10 points in the PF-10 was defined based on a distribution-based method by Cohen [13]. The primary endpoint of the OTIVACTO study was the occurrence of therapeutic success, defined as a 10-point increase in the PF-10 score, between visit 1 (baseline) and visit 2 (approximately 6 weeks after starting treatment) [10] based on the experience in two 1-year studies conducted in COPD patients [14]. In this analysis, we extracted the data of the Swiss patient group and compared the results to the rest of the cohort.

The study was carried out in compliance with the protocol, the principles laid down in the Declaration of Helsinki, and as close as possible to the standards of the International Conference of Harmonization Tripartite Guideline for Good Clinical Practice, Guidelines for Good Epidemiological Practice and Good Pharmacoepidemiology Practice. The sponsor of the OTIVACTO ${ }^{\circledR}$ trial (Boehringer Ingelheim) is committed to responsible sharing of clinical study reports, related clinical documents, and patient-level clinical study data. Researchers are invited to submit inquiries via the Clinical Study Data Request website (https://www.clinicalstudydatarequest.com). This study was approved by the Swiss Ethics Committee (BASEC Nr.: 2016-01156) and was registered at the Clinical Trials Registry (NCT02720757).

\section{Statistical analysis}

A change of 10 in the PF-10 score between visits was considered as a therapy success and was used to differentiate between responders and non-responders. The correlation between the change in PF-10 and certain baseline characteristics were analyzed by means of Pearson correlation coefficients.

To compare patient subgroups, $\mathrm{P}$ values for continuous variables were calculated using Wilcoxon rank sum test (Mann-Whitney U test). Categorical variables were compared by $\chi^{2}$ test. If $\chi^{2}$ test was not valid, the comparison was done using Fisher's exact test. A P $<0.05$ was considered significant. All analyses are exploratory and $\mathrm{P}$ values are descriptive. All calculations were performed using the statistical analysis system SAS version 9.4.

\section{Results}

The total cohort consisted of 7,608 participants, of whom 94 
were from Switzerland, and 7,514 were from eastern European countries. The baseline characteristics are listed in Table 1. The Swiss participants were significantly older, consisted of more female patients, had significantly more comorbidities and had lower COPD stages as compared to the total cohort. Regarding the other demographical data, the Swiss subgroup of patients did not differ from the total cohort.

Overall, 110 drug-related adverse events were reported for the treated set population [10] with no significant differences between the Swiss and the other European countries.

There was no significant difference in the response to the fixed combination Tio/Olo between the participants of both groups (Switzerland 63\% versus other countries 67\%). Regarding the age groups, the responder and non-responder rate in the Swiss group $\geq 65$ years was significant higher than in other countries $(75 \%$ vs. $54 \%$; $\mathrm{P}=0.0197)$ for the responder group and $77 \%$ vs. $54 \%(\mathrm{P}=0.0069)$ for the non-responder group.

According to the COPD stages, no significant differences were found regarding the non-responder and responder rate between the Swiss group and other countries.

Also in the subgroup analyses with regard to age, number of comorbidities, dyspnea symptoms measured in $\mathrm{mMRC}$, COPD stages and smoker status, there were no significant differences between the Swiss group and the other countries. These results are shown in Table 2.

Figures 1 and 2 show the positive correlation between the mMRC score and subjective response to the medication for both groups. The more dyspnea complaints, the higher the subjective improvement following implementation of Tio/Olo.

There was no correlation found between the self-reported exacerbation frequency and response to the medication shown in Figure 3 for the Swiss cohort but for the other countries (Fig. 4).

A significant positive correlation was found between the score in the mMRC questionnaire and the changes in the PF10 for both groups. This was also true for the exacerbation rate and the PF-10 for the European countries group but not for the Switzerland group.

Tables 3 and 4 provide the Pearson (partial) correlation analysis for Switzerland and the other countries showing no influence of the age differences and the comorbidities on the results in the PF-10 and $\mathrm{mMRC}$ score.

\section{Discussion}

This analysis shows that despite country-specific differences in the health care systems in the participating countries, especially in Switzerland, the results on the response to the fixed combination Tio/Olo in COPD were similar to findings from the overall cohort. A comparison of research results between various health services, socio-economic and cultural contexts is worthwhile because the conditions and circumstances under which research have been conducted are quite different. For example, weight loss in COPD appears to affect many patients (from $25 \%$ to $35 \%$ in several series), at least in Europe and North America. However, the situation is thought to be different in the Mediterranean area [15]. The implications of these diversities for the therapeutic approach remain unclear. However, in this analysis, we could not find any major differences between the results from Switzerland and those from a number of eastern European countries. The results were comparable throughout. Interestingly, Swiss participants were on average older and showed significantly more comorbidities. This might be explained by overall higher life expectancy in Switzerland, shifting the age groups or later onset of smoking as potential reasons for this observation.

Despite the aforementioned differences in baseline characteristics, there were no significant differences in the subgroup analyses. The relationship between responder and nonresponder was almost identical in all groups. Smoking status, age and the presence of cardiac comorbidities did not have an impact on therapeutic success, confirming that bronchodilators are effective treatments irrespective of patient baseline characteristics. Recent findings indicate that effective bronchodilation in COPD patients can improve cardiac function mainly by reducing hyperinflation, independently supporting an early start of optimal bronchodilation in COPD [16]. Nevertheless, physical comorbidities such as obesity, musculoskeletal and neurological conditions in people with COPD reduce physical activity. Physical activity is an important determinant of morbidity and mortality in people with COPD. Significantly less time is spent performing light and moderate intensity physical activity and more time is spent engaged in sedentary behavior compared with people with COPD without physical comorbidities and healthy people. Low levels of self-reported physical activity are related to an increase in hospitalizations and a higher risk of death. Reduced physical activity has also been associated with greater disease severity in COPD, higher levels of dyspnea, lower exercise capacity and poorer healthrelated quality of life.

Previous studies indicate that the occurrence of exacerbations varies widely, and that FEV1 by itself is not sufficient to predict exacerbations in COPD [17]. In this context, it is known that symptoms in COPD may have a greater impact on patients' functional performance than lung function documented by the stronger correlation between functional performance inventory short form (FPI-SF) and COPD assessment test (CAT) scores compared to FPI-SF and FEV1 $\%$ predicted [18]. It has also been shown that there is an association between symptoms and a patient's physical activity level and therefore improving 24-h symptoms should be an important consideration in the management of COPD [19]. In the present study, no significant differences were found between the different stages of COPD in terms of response to the medication. The relationship between responder and non-responder was identical in all stages, confirming that FEV1 is an unreliable parameter in terms of medication response.

In their latest guidelines, the American Respiratory Society (ATS) recommends LABA/LAMA combination therapy in patients with COPD who complain of dyspnea or exercise intolerance [20]. In consequence, pharmacological therapy in COPD might reduce total time spent in sedentary behavior due to variety of mechanisms, such as improvements in breathlessness, static and dynamic hyperinflation, breathing effort, cardiovascular function, and/or exercise capacity. The current 
Table 1. Demographic Data and Baseline Characteristics

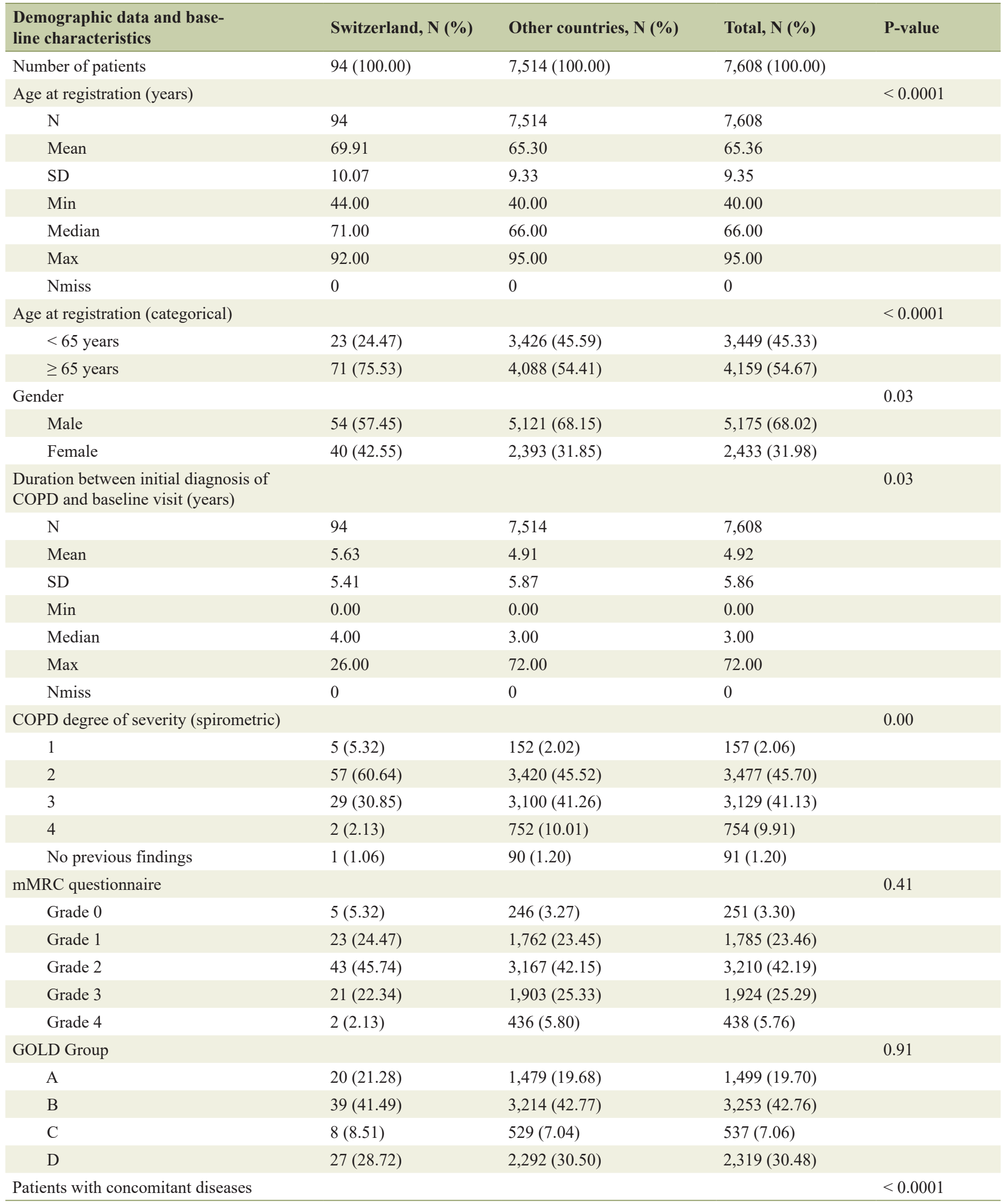


Table 1. Demographic Data and Baseline Characteristics (continued)

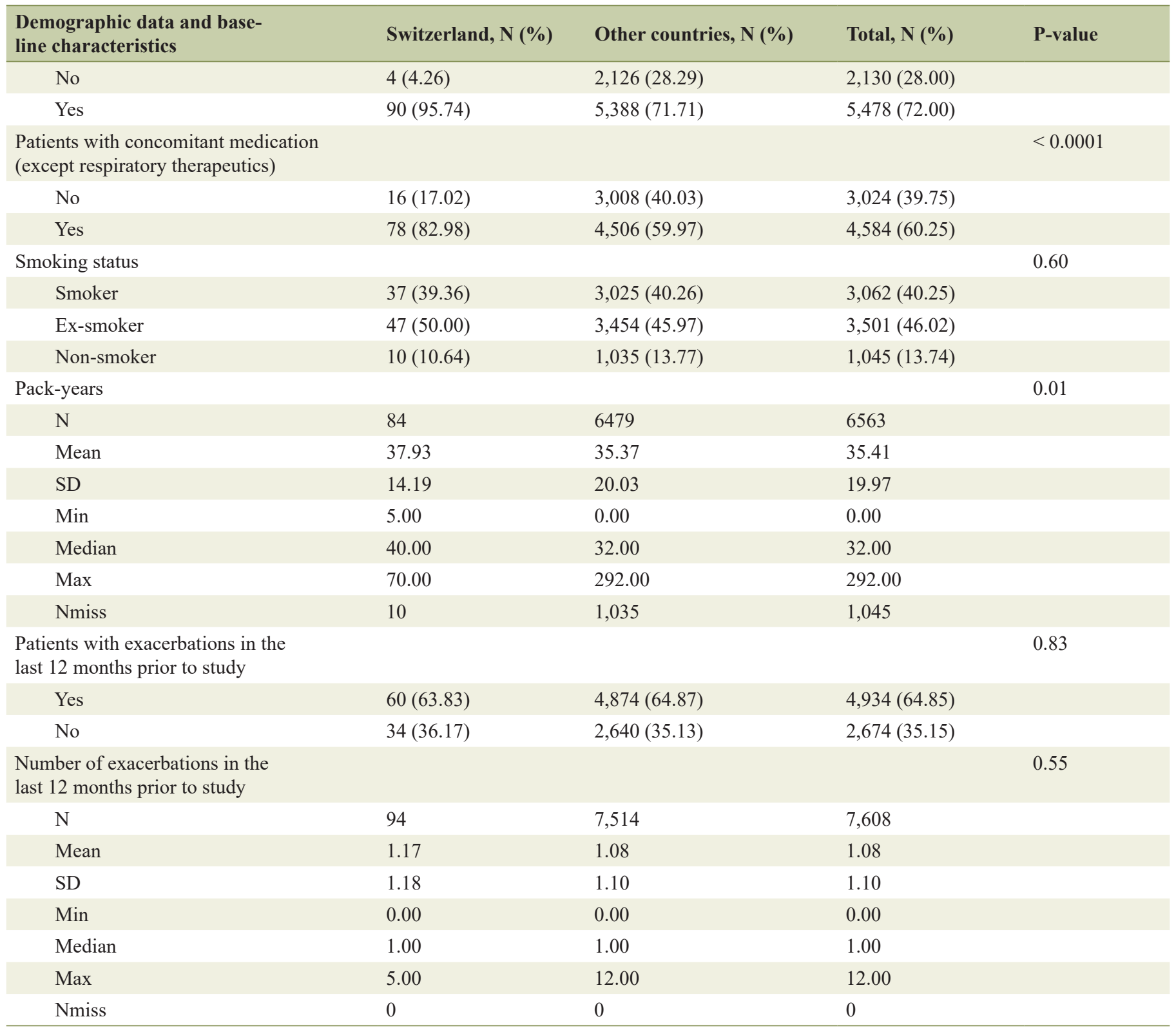

COPD: chronic obstructive pulmonary disease; SD: standard deviation; mMRC: modified Medical Research Council questionnaire; Nmiss: number missings.

data confirm this strategy using Tio/Olo as an LAMA/LABA combination in a large proportion to improve their ability to carry out daily activities resulted in high patient satisfaction with the treatment. However, in this context, it is important to keep in mind that subjective physical activity is limited in correlation with measured physical activity. Though subjective methods are particularly suitable for large population surveys, they are susceptible to recall bias [21]. However, LAMA/ LABA fixed-dose combination in COPD improves lung function and measures of disease worsening, if compared to monobronchodilation or LABA/ICS therapy [22-24].

In this analysis, we observed a positive correlation for symptomatic patients, as shown in the mMRC score. As already shown in other studies with different therapeutic interventions, the patients with most symptoms are those who also benefit most from a medical treatment [25]. In our analysis, those with a high mMRC score are most likely to benefit from the pharmacological intervention. The $\mathrm{mMRC}$ grade $\geq 2$ can predict a low physical activity level. Therefore, assessment of breathlessness by the mMRC questionnaire as already recommended by the ATS would be useful to stratify the risks of reduced physical activity in COPD and may have an influence on deciding the pharmacotherapy strategy [26]. Surprisingly we found no correlation between self-reported exacerbation and 


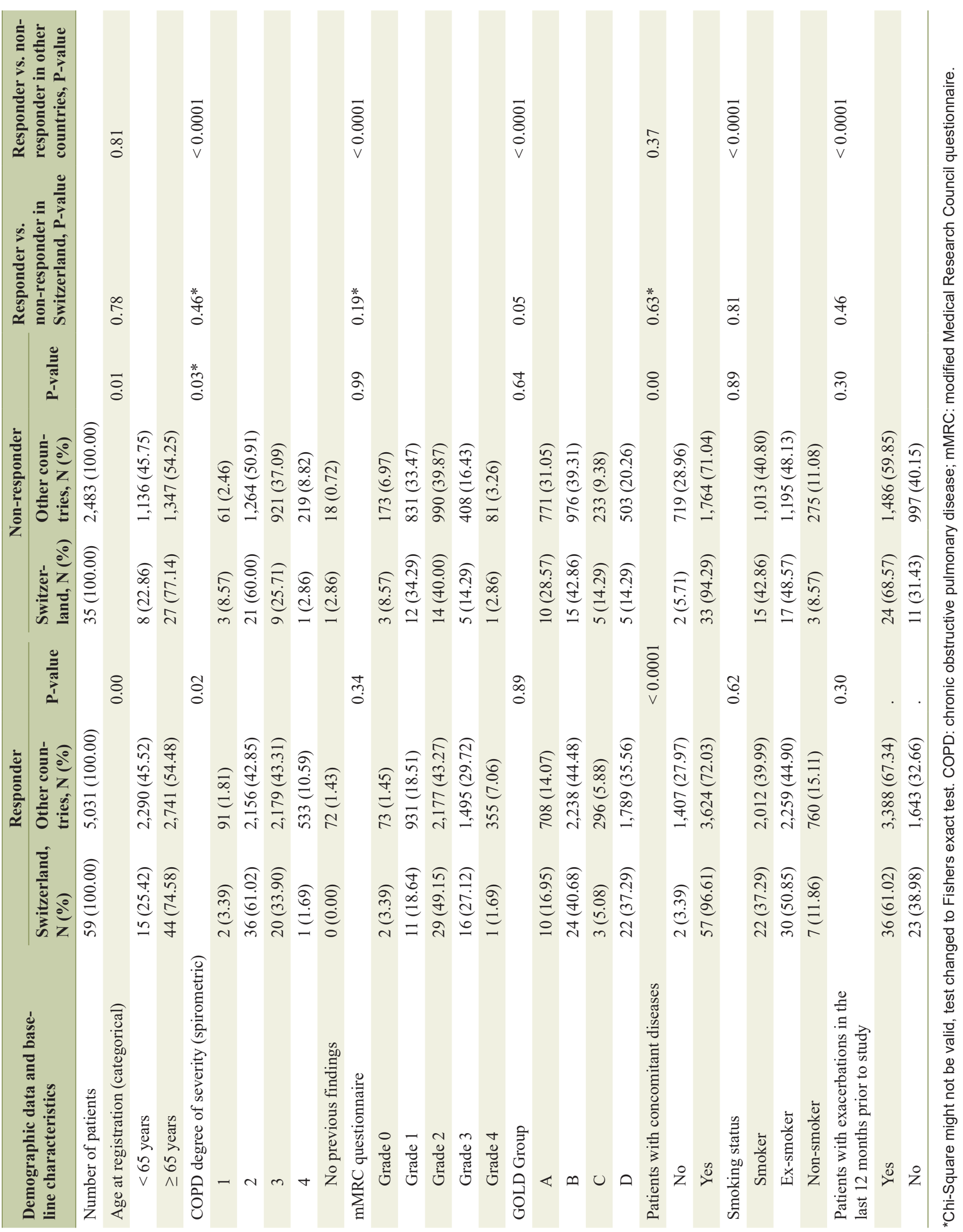




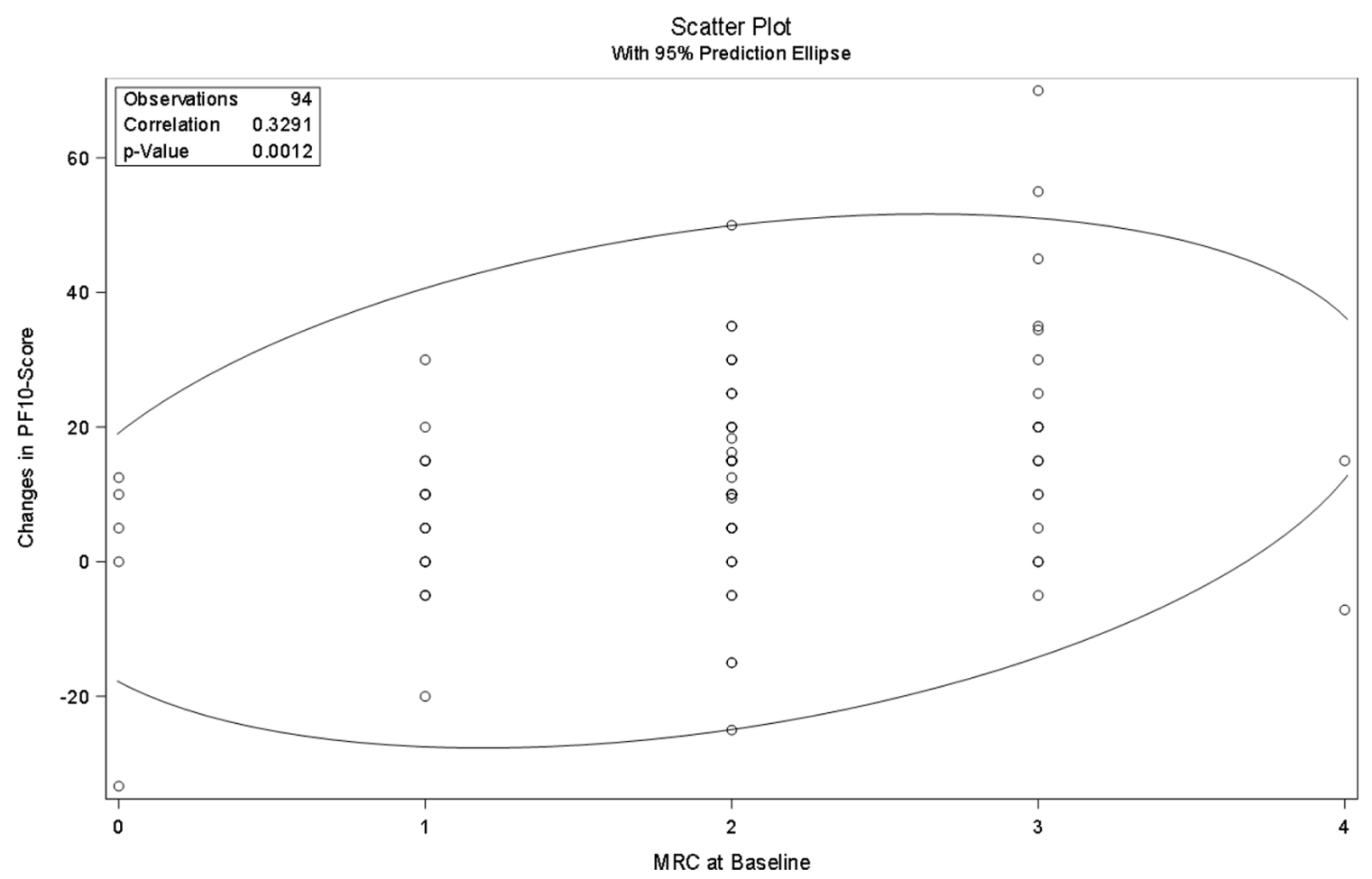

Figure 1. Pearson correlation analysis of change in PF-10 and mMRC in Switzerland. PF-10: 10-question physical functioning questionnaire; mMRC: modified Medical Research Council questionnaire.

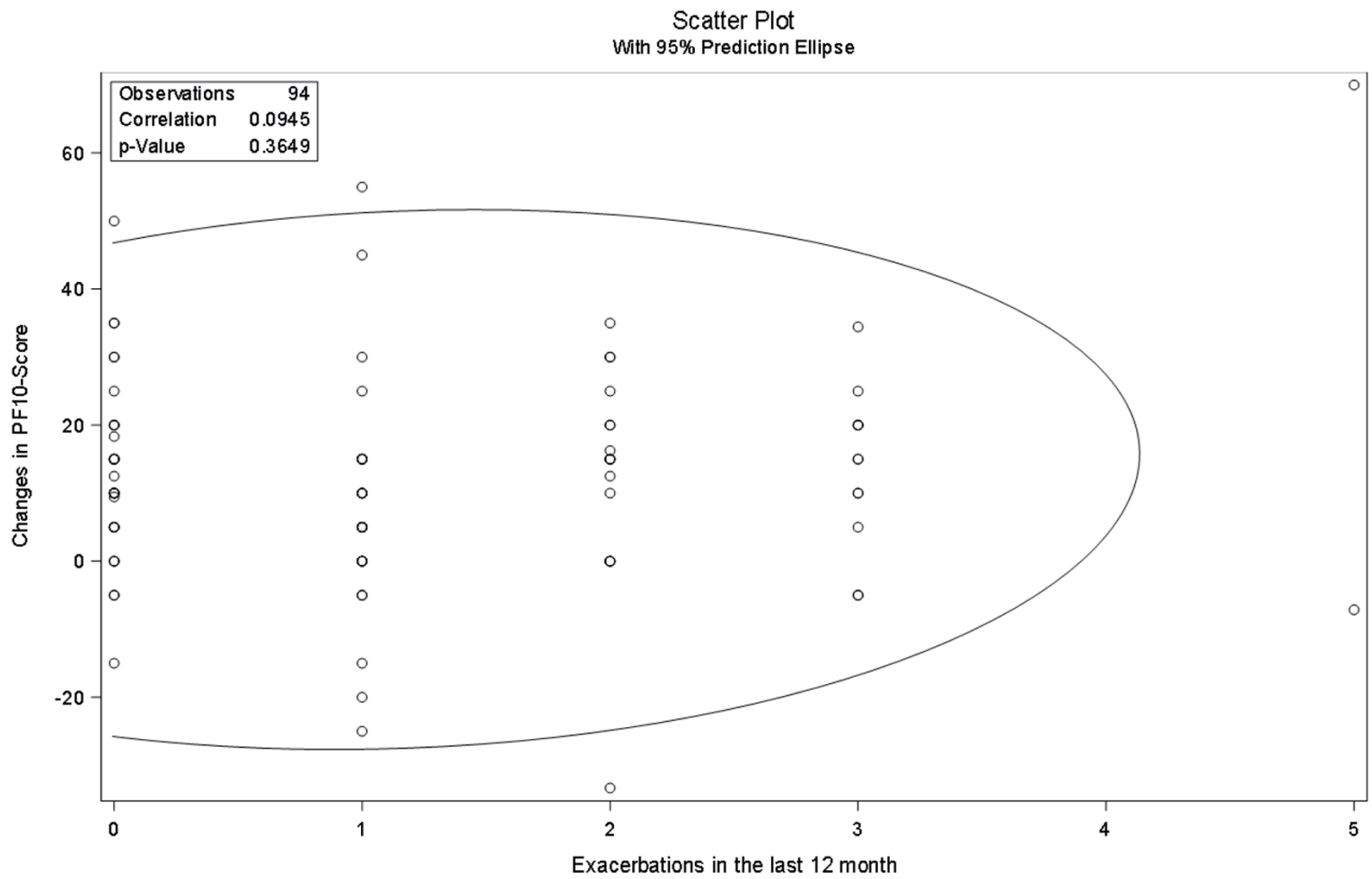

Figure 2. Pearson correlation analysis of change in PF-10 and exacerbations in the last 12 months in Switzerland. PF-10: 10-question physical functioning questionnaire. 


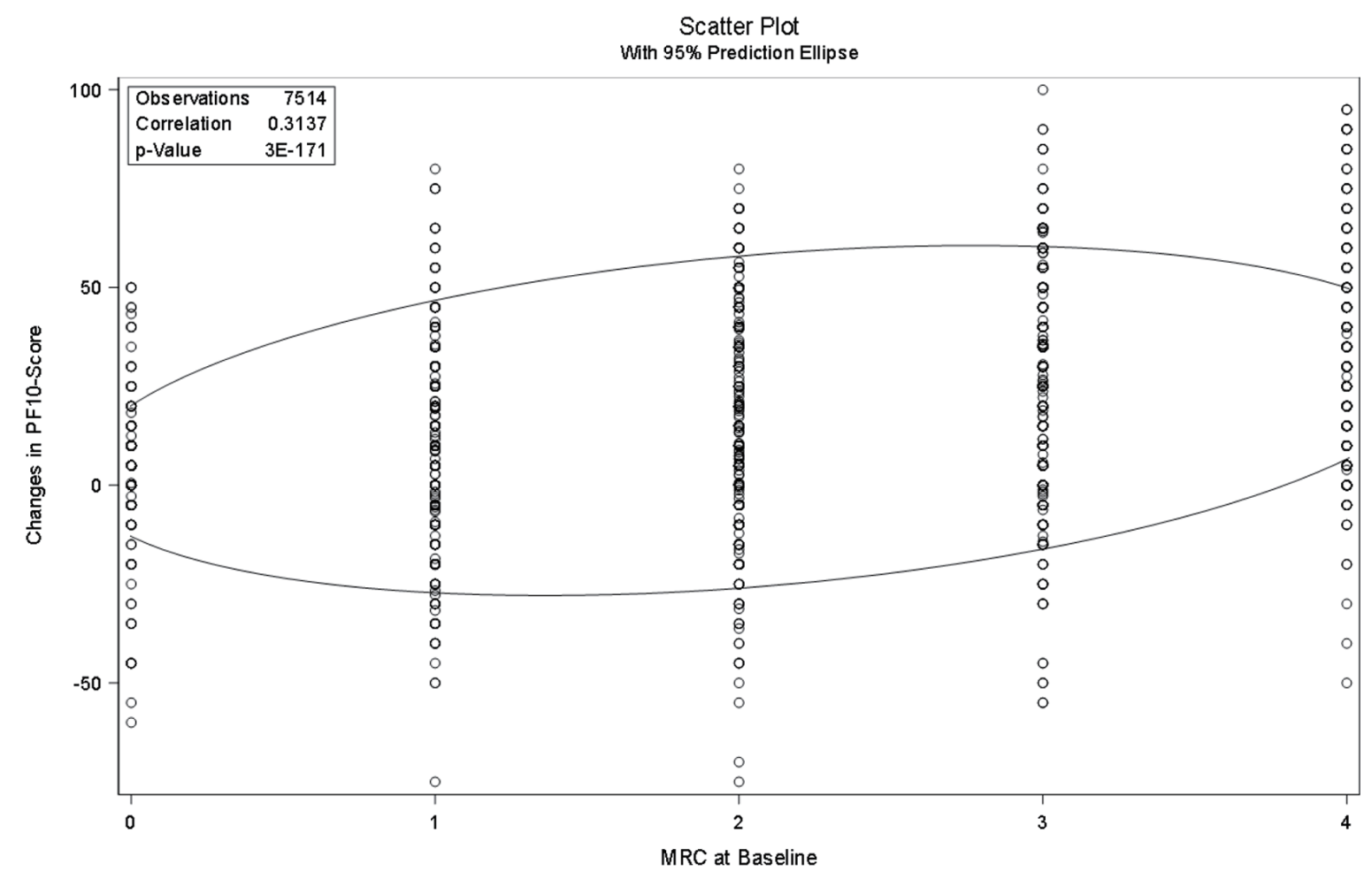

Figure 3. Pearson correlation analysis of change in PF-10 and mMRC in other countries. PF-10: 10-question physical functioning questionnaire; mMRC: modified Medical Research Council questionnaire.

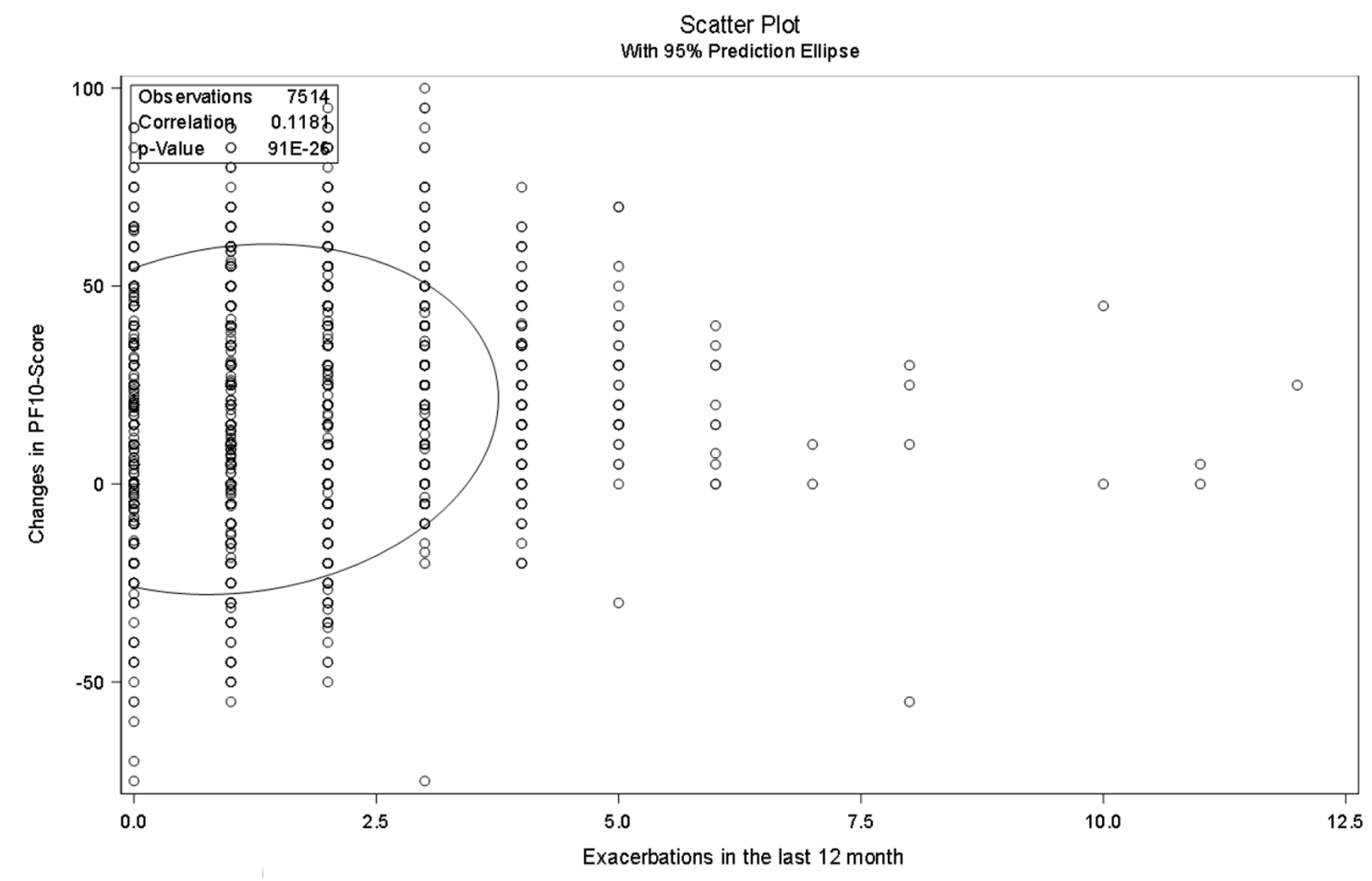

Figure 4. Pearson correlation analysis of change in PF-10 and exacerbations in the last 12 months in other countries. PF-10: 10-question physical functioning questionnaire. 
Table 3. Pearson (Partial) Correlation Analysis in Switzerland $(N=94)$

\begin{tabular}{lll}
\hline Changes in PF-10 score & mMRC & Number of exacerbations \\
\hline Nothing & & 0.0945 \\
$\quad$ Correlation coefficient & 0.33 & 0.3649 \\
$\quad$ P-value & 0.00 & 0.0948 \\
Age (<65 vs. $\geq 65)$ & 0.35 & 0.3660 \\
$\quad$ Correlation coefficient & 0.00 & 0.0931 \\
$\quad$ P-value & & 0.3748 \\
Concomitant disease (yes vs. no) & 0.33 & 0.0937 \\
$\quad$ Correlation coefficient & 0.00 & 0.3744 \\
$\quad$ P-value & & 0.35 \\
Age (<65 vs. $\geq 65)$ and concomitant disease (yes vs. no) & 0.00 & \\
$\quad$ Correlation coefficient & & \\
P-value & & 0.35 \\
\hline
\end{tabular}

PF-10: 10-question physical functioning questionnaire; mMRC: modified Medical Research Council questionnaire.

enhancement in the PF-10 score in the Swiss group. Regarding the total cohort, therapeutic success was found in $64.3 \%$ (95\% confidence interval (CI) $63.0-65.6 \%$ ) of patients with infrequent $(\leq 1)$ and $76.1 \%(95 \%$ CI $74.3-77.9 \%)$ of patients with frequent $(\geq 2)$ exacerbations $(\mathrm{P}<0.0001)$ [10], which was also confirmed by other studies showing that dual bronchodilation has an influence on COPD exacerbations [27, 28]. We suspect a selection bias regarding this result. While in Switzerland mainly clinics recruited the participants, in Eastern Europe mainly GPs and resident pneumologists included patients in the trial. This assumption is confirmed by the fact that the Swiss participants showed significant more comorbidities and were therefore more impaired leading to less perception of improvements.

There are some limitations to be discussed. First, the main limitation of this analysis is that this study was designed without having a comparison group to detect treatment efficacy in COPD as the primary effectiveness outcome in the overall population. However, this is a fundamental problem of noninterventional studies. Nevertheless, the results of our study seem to confirm the results of other studies and probably go beyond a simple placebo effect $[29,30]$. We also conducted this analysis to show that the results of the overall cohort are also transferable to the Swiss population. Second, the group of Swiss participants is relatively small and was included in the study by clinicians. However, we consider the findings sufficiently valid with the exception of the correlation between exacerbation frequency and success regarding the PF-10 score as already described. Third, although an actual measurement of physical activity would have been more meaningful for the participants, questionnaires like the PF-10 provide good correlations to actual physical activity in large populations. It should be noted that the subjective data in PF-10 correlated well with the physical activity, at least in comparative studies

Table 4. Pearson (Partial) Correlation Analysis in Other Countries $(\mathrm{N}=7,514)$

\begin{tabular}{|c|c|c|}
\hline Changes in PF-10 score & mMRC & Number of exacerbations \\
\hline \multicolumn{3}{|l|}{ Nothing } \\
\hline Correlation coefficient & 0.31 & 0.1181 \\
\hline \multicolumn{3}{|l|}{ Age $(<65$ vs. $\geq 65)$} \\
\hline Correlation coefficient & 0.32 & 0.1183 \\
\hline \multicolumn{3}{|c|}{ Concomitant disease (yes vs. no) } \\
\hline Correlation coefficient & 0.32 & 0.1193 \\
\hline P-value & $<0.00$ & $<0.0001$ \\
\hline \multicolumn{3}{|c|}{ Age $(<65$ vs. $\geq 65)$ and concomitant disease (yes vs. no) } \\
\hline Correlation coefficient & 0.32 & 0.1197 \\
\hline
\end{tabular}

PF-10: 10-question physical functioning questionnaire; mMRC: modified Medical Research Council questionnaire. 
in different patient groups [31, 32]. Fourth, the study was carried out in a short period of time, so longer lasting therapeutic effects could not be assessed.

The strength of this study is the large representative sample size. Furthermore, this study was carried out in a realworld primary care setting and represented a wide spectrum of COPD severities, background treatments, and comorbidities, thus providing valuable data and insights reflecting clinical practice in European countries, including Switzerland.

\section{Conclusion}

This analysis suggests that the treatment with fixed bronchodilation was able to enhance the physical functioning in COPD patients in their daily lives, and importantly, there was no difference between the Swiss and the total cohort. Maintenance treatment in COPD patients with tiotropium/olodaterol helped patients to remain active especially for those with more dyspnea symptoms.

\section{Acknowledgments}

None to declare.

\section{Financial Disclosure}

Dr. Marc Spielmanns and Dr. Michael Tamm report personal fees and non-financial support from Boehringer Ingelheim. Dr. Sebastian Schildge is employee of Boehringer Ingelheim. Dr. Arschang Valipour reports personal fees, non-financial support from Boehringer Ingelheim, personal fees from Novartis, Chiesi, and AstraZeneca, during the conduct of the study.

\section{Conflict of Interest}

None to declare.

\section{Informed Consent}

Not applicable.

\section{Author Contributions}

The authors meet criteria for authorship as recommended by the International Committee of Medical Journal Editors. They take full responsibility for the scope, direction, content of, and editorial decisions relating to, the manuscript, were involved at all stages of development, and have approved the submitted manuscript. All authors contributed to data analysis, drafting and revising the article, gave final approval of the version to be published, and agree to be accountable for all aspects of the work.

\section{Data Availability}

The authors declare that data supporting the findings of this study are available within the article.

\section{References}

1. Vogelmeier CF, Criner GJ, Martinez FJ, Anzueto A, Barnes PJ, Bourbeau J, Celli BR, et al. Global strategy for the diagnosis, management, and prevention of chronic obstructive lung disease 2017 report: GOLD executive summary. Arch Bronconeumol. 2017;53(3):128-149.

2. GBD Chronic Respiratory Disease Collaborators. Prevalence and attributable health burden of chronic respiratory diseases, 1990-2017: a systematic analysis for the Global Burden of Disease Study 2017. Lancet Respir Med. 2020;8(6):585-596.

3. Antonelli-Incalzi R, Imperiale C, Bellia V, Catalano F, Scichilone N, Pistelli R, Rengo F, et al. Do GOLD stages of COPD severity really correspond to differences in health status? Eur Respir J. 2003;22(3):444-449.

4. Casanova C, Marin JM, Martinez-Gonzalez C, de LucasRamos P, Mir-Viladrich I, Cosio B, Peces-Barba G, et al. Differential effect of modified medical research council dyspnea, COPD assessment test, and clinical COPD questionnaire for symptoms evaluation within the new GOLD staging and mortality in COPD. Chest. 2015;148(1):159168 .

5. Global Initiative for Chronic Obstructive Lung Disease. Global strategy for the diagnosis, management, and prevention of chronic obstructive pulmonary disease: 2020 report. Available from: https:/goldcopd.org/gold-reports/. Accessed July 17, 2020.

6. O'Donnell DE, Casaburi R, Frith P, Kirsten A, De Sousa D, Hamilton A, Xue W, et al. Effects of combined tiotropium/ olodaterol on inspiratory capacity and exercise endurance in COPD. Eur Respir J. 2017;49(4):1601348.

7. Troosters T, Maltais F, Leidy N, Lavoie KL, Sedeno M, Janssens W, Garcia-Aymerich J, et al. Effect of bronchodilation, exercise training, and behavior modification on symptoms and physical activity in chronic obstructive pulmonary disease. Am J Respir Crit Care Med. 2018;198(8):1021-1032.

8. Thomas M, Decramer M, O'Donnell DE. No room to breathe: the importance of lung hyperinflation in COPD. Prim Care Respir J. 2013;22(1):101-111.

9. Maltais F, O'Donnell D, Galdiz Iturri JB, Kirsten AM, Singh D, Hamilton A, Tetzlaff K, et al. Effect of 12 weeks of once-daily tiotropium/olodaterol on exercise endurance during constant work-rate cycling and endurance shuttle walking in chronic obstructive pulmonary disease. Ther Adv Respir Dis. 2018;12:1753465818755091.

10. Valipour A, Tamm M, Kocianova J, Bayer V, Sanzharovskaya M, Medvedchikov A, Haaksma-Herczegh M, et al. Improvement in self-reported physical functioning with tiotropium/olodaterol in central and eastern European COPD Patients. Int J Chron Obstruct Pulmon Dis. 2019;14:2343- 
2354.

11. Miravitlles M, Vogelmeier C, Roche N, Halpin D, Cardoso J, Chuchalin AG, Kankaanranta H, et al. A review of national guidelines for management of COPD in Europe. Eur Respir J. 2016;47(2):625-637.

12. Ware JE, Jr., Sherbourne CD. The MOS 36-item shortform health survey (SF-36). I. Conceptual framework and item selection. Med Care. 1992;30(6):473-483.

13. Cohen J, editor. Statistical power analysis for the behavioral sciences. 1st ed. Hillsdale (NJ): Lawrence Erlbaum Associates Inc.; 1977.

14. Rau-Berger H, Mitfessel H, Glaab T. Tiotropium Respimat $(\mathrm{R})$ improves physical functioning in chronic obstructive pulmonary disease. Int J Chron Obstruct Pulmon Dis. 2010;5:367-373.

15. Coronel C, Orozco-Levi M, Ramirez-Sarmiento A, Martinez-Llorens J, Broquetas J, Gea J. [Low-weight syndrome associated with COPD in our setting]. Arch Bronconeumol. 2002;38(12):580-584.

16. Hohlfeld JM, Vogel-Claussen J, Biller H, Berliner D, Berschneider K, Tillmann HC, Hiltl S, et al. Effect of lung deflation with indacaterol plus glycopyrronium on ventricular filling in patients with hyperinflation and COPD (CLAIM): a double-blind, randomised, crossover, placebo-controlled, single-centre trial. Lancet Respir Med. 2018;6(5):368-378.

17. Huang WC, Wu MF, Chen HC, Hsu JY, Taiwan Obstructive Lung Disease C. Characteristics and risk factors for inconsistency between the risk of exacerbations and the severity of airflow limitation in COPD based on GOLD 2017: A retrospective, cross-sectional study. PLoS One. 2018;13(3):e0193880.

18. Ding B, Judge D, Small M, Bent-Ennakhil N, Siddiqui S. Functional performance in patients with COPD: association with treatment regimen, GOLD group, lung function, and symptom burden in a cross-sectional study. Int J Chron Obstruct Pulmon Dis. 2018;13:2785-2796.

19. Miravitlles M, Worth H, Soler Cataluna JJ, Price D, De Benedetto F, Roche N, Godtfredsen NS, et al. Observational study to characterise 24-hour COPD symptoms and their relationship with patient-reported outcomes: results from the ASSESS study. Respir Res. 2014;15:122.

20. Nici L, Mammen MJ, Charbek E, Alexander PE, Au DH, Boyd CM, Criner GJ, et al. Pharmacologic management of chronic obstructive pulmonary disease. An official American thoracic society clinical practice guideline. Am J Respir Crit Care Med. 2020;201(9):e56-e69.

21. Muller C, Winter C, Rosenbaum D. Current objective techniques for physical activity assessment in comparison with subjective methods. Dtsch Z Sportmed. 2010;61(1):11-18.

22. Anzueto AR, Vogelmeier CF, Kostikas K, Mezzi K, Fucile S, Bader G, Shen S, et al. The effect of indacaterol/glycopyrronium versus tiotropium or salmeterol/fluticasone on the prevention of clinically important deterioration in COPD. Int J Chron Obstruct Pulmon Dis. 2017;12:1325-1337.

23. Greulich T, Kostikas K, Gaga M, Aalamian-Mattheis M, Lossi NS, Patalano F, Nunez X, et al. Indacaterol/glycopyrronium reduces the risk of clinically important deterioration after direct switch from baseline therapies in patients with moderate COPD: a post hoc analysis of the CRYSTAL study. Int J Chron Obstruct Pulmon Dis. 2018;13:12291237.

24. Anzueto AR, Kostikas K, Mezzi K, Shen S, Larbig M, Patalano F, Fogel R, et al. Indacaterol/glycopyrronium versus salmeterol/fluticasone in the prevention of clinically important deterioration in COPD: results from the FLAME study. Respir Res. 2018;19(1):121.

25. Spielmanns M, Gloeckl R, Schmoor C, Windisch W, Storre JH, Boensch M, Kenn K. Effects on pulmonary rehabilitation in patients with COPD or ILD: A retrospective analysis of clinical and functional predictors with particular emphasis on gender. Respir Med. 2016;113:8-14.

26. Hayata A, Minakata $Y$, Matsunaga K, Nakanishi M, Yamamoto N. Differences in physical activity according to mMRC grade in patients with COPD. Int J Chron Obstruct Pulmon Dis. 2016;11:2203-2208.

27. Ariel A, Altraja A, Belevskiy A, Boros PW, Danila E, Flezar M, Koblizek V, et al. Inhaled therapies in patients with moderate COPD in clinical practice: current thinking. Int J Chron Obstruct Pulmon Dis. 2018;13:45-56.

28. Beeh KM, Burgel PR, Franssen FME, Lopez-Campos JL, Loukides S, Hurst JR, Flezar M, et al. How Do Dual LongActing Bronchodilators Prevent Exacerbations of Chronic Obstructive Pulmonary Disease? Am J Respir Crit Care Med. 2017;196(2):139-149.

29. Steinmetz KO, Abenhardt B, Pabst S, Hansel M, Kondla A, Bayer V, Buhl R. Assessment of physical functioning and handling of tiotropium/olodaterol Respimat((R)) in patients with COPD in a real-world clinical setting. Int J Chron Obstruct Pulmon Dis. 2019;14:1441-1453.

30. Molina Paris J, Alonso Hernandez PM, Diez Garcia JA, Gonzalez Uribe-Etxebarria I, Yelo Garcia J, Galera Llorca $\mathrm{J}$, Aguilar $\mathrm{H}$, et al. Assessment of physical functioning in patients with chronic obstructive pulmonary disease (COPD) requiring long-acting dual bronchodilation in routine clinical practice. Semergen. 2020;S1138-3593(20):30402-0.

31. ten Klooster PM, Oude Voshaar MA, Gandek B, Rose M, Bjorner JB, Taal E, Glas CA, et al. Development and evaluation of a crosswalk between the SF-36 physical functioning scale and Health Assessment Questionnaire disability index in rheumatoid arthritis. Health Qual Life Outcomes. 2013;11:199.

32. Glaab T, Vogelmeier C, Buhl R. Outcome measures in chronic obstructive pulmonary disease (COPD): strengths and limitations. Respir Res. 2010;11:79. 